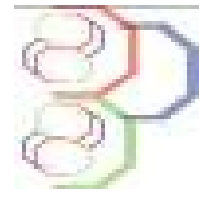

Journal of Applied Biosciences 69:5510 - 5522

ISSN 1997-5902

\title{
Biodiversité et valeur alimentaire des fruits au Cameroun : observations préliminaires dans le Département de la Bénoué (Région du Nord)
}

Kouebou ${ }^{124^{*}}$ C., Fadi Goygoy¹, Bourou² S., Kosga Djakissam² P., Layla H. ${ }^{1}$, Zenabou¹ G., Barbi ${ }^{1}$ M., Vunyingah ${ }^{3}$ M., Woin ${ }^{2}$ N.

${ }^{1}$ Institut de Recherche Agricole pour le Développement (IRAD)-Programme Technologie Alimentaire et Post-Récolte BP 415, Garoua, Cameroun ;

2 IRAD - Conservatoire fruitier de Kismatari BP 415, Garoua, Cameroun;

3 IRAD - Programme socio-économie BP 415, Garoua, Cameroun;

${ }^{4}$ Central Africa Food Data System (CAFOODS) for AFROFOODS/INFOODS/FAO

* correspondance : kchristiant@yahoo.fr

Original submitted in on $21^{\text {st }}$ February 2013 Published online at www.m.elewa.org on 30 ${ }^{\text {th }}$ September 2013.

https://dx.doi.org/10.4314/jab.v69i0.95077

\section{RÉSUMÉ}

Introduction : La promotion de l'arboriculture fruitière (familiale ou industrielle) est une des priorités de la Recherche et Développement agricole au Cameroun. Cependant, faute de statistiques fiables, elle semble méconnue et sous-estimée notamment dans les zones semi-arides en proie à une insécurité environnementale et alimentaire récurrente. Cette situation limite les perspectives de valorisation des ressources fruitières et justifie l'initiation de la présente étude dont l'objectif est d'apprécier la biodiversité et la valeur alimentaire des fruits disponibles au (nord-) Cameroun.

Méthodologie et Résultat: Une synthèse documentaire couplée à des entretiens avec des personnes ressources et des observations directes ont permis de répertorier la biodiversité disponible à l'Institut de Recherche Agricole pour le développement (IRAD) et de caractériser les ventes dans 7 marchés de l'Arrondissement de Garoua (Département de la Bénoué, Région du Nord) pendant la saison des pluie. L'échantillonnage des fruits a été conduit sur ces marchés en saison de pluie (Juillet à Septembre) et leurs caractéristiques physico-sensorielles et marchandes ont été appréciées. II ressort que le conservatoire de I'IRAD de Garoua entretient majoritairement 17 espèces d'agrumes (122 variétés) et 71 variétés de manguiers (308 pieds en somme). Sur les marchés de Garoua, une vingtaine de fruits a été répertoriée avec huit fruits dominant les expositions-ventes : l'avocat, la banane, l'orange, le fruit du karité, la mangue, le tamarin, le baobab et le citron. Leur identité botanique a été révélée. L'examen de leurs propriétés physico-sensorielles et marchandes confirme l'étendue de cette biodiversité mais surtout son accessibilité à travers des prix allant de 10 à 1300 Francs CFA/fruit.

Conclusion et application : Au regard des multiples atouts des fruits, il est envisagé d'étendre les observations à d'autres périodes et zones climatiques. Ceci devrait contribuer à une meilleure de connaissance de l'offre et de la consommation fruitière au (nord-) Cameroun. Compte tenu de leur richesse en nutriments, l'incorporation de ces fruits et/ou de leurs dérivés dans l'alimentation est encouragée en faveur d'une nutrition saine dans les zones de savanes marquée par des régimes carencés en micronutriments. Les voies de valorisation via la transformation locale devraient être explorées en faveur d'une meilleure conservation des fruits. 
Mots clés : Biodiversité, Fruits, Commerce, Savane

Fruit diversity and value in Cameroon: preliminary observations in Benoue Division (North Region)

Abstract

Introduction: The promotion of fruit tree farming for household or industrial purpose constitutes one of the priorities of agricultural research and development in Cameroon. However, the lack of reliable statistics, leads to underestimation of fruit value especially in semi-arid areas affected by recurrent environmental stress and food insecurity. This limits the prospects of development of fruit resources and justifies the initiation of this study aimed to assess the biodiversity and food value of fruit available in (North) Cameroon.

Methodology and Results A literature review coupled with interviews involving resourceful persons and direct observations helped to classify the fruit biodiversity nature at the Institute of Agricultural Research for Development (IRAD) and to characterize sales in seven markets of Garoua (Benoue Division, North Region). Fruit sampling was conducted at these markets during the rainy season (July to September) and their physicosensory characteristics and commercial value assessed. It appears that the fruit conservatory unit of IRAD Garoua harbors principally 17 citrus fruit species (122 varieties) and 71 varieties of mango (308 trees in totals). At Garoua, market 21 fruits were classified with eight fruits dominating the sales: avocados, banana, orange, Shea fruit, mango, tamarind, baobab, and lemon. Their botanical identity was revealed and the examination of their physico-sensory properties and commercial value confirms the extent of biodiversity but also their affordability through prices ranging from 10 to $1,300 \mathrm{CFA}$ / fruit.

Conclusion and application: In view of the many advantages of fruits, it is proposed to extend the observations to other periods and climatic zones. This should contribute to a better knowledge of the supply and fruit consumption in (North) Cameroon. Due to their high nutrient value, the consumption of these fruits and products is strongly encouraged for a healthy nutrition in savannah areas in prey of micronutrient deficiencies. Valorization approaches through local processing need to be explored for a better preservation of fruit diversity. Keywords: Biodiversity, Fruits, Trade, Savannah.

\section{INTRODUCTION}

Un consensus émerge en faveur de l'amélioration de la consommation des fruits à travers la promotion de l'arboriculture fruitière mais également à partir des produits de cueillette largement répandue chez les populations subsahariennes (Normand, 1992; Arbonnier, 2000 ; Bâ et al., 2000 ; De Caluwé et al., 2010). En zones rurale et urbaine, les fruits « sauvages " occupent une part importante de l'alimentation et leur contribution à la survie des populations est indéniable en période de crise (soudure, inondation, sècheresse, famine, conflit politico-militaire.) (Grivetti, 1978; Ogle \& Grivetti, 1985; Humphrey et al., 1993 ; Herzorg et al., 1994 ; Smith \& Grivetti, 1994; Eyog et al., 2006). Les fruits et dérivés sont une source importante de nutriments et d'autres composés bioactifs aux potentialités multiformes. Ils contiennent à des teneurs variables en eau (15,9 à 90,2 g/100 $\mathrm{g}$ de portion comestible selon le fruit), en minéraux $(0,02$ à $4,3 \mathrm{~g} / 100 \mathrm{~g})$ ou en vitamine $C(0,07$ to $247 \mathrm{mg} / 100 \mathrm{~g})$ tel que le précise FAO-IN-FOODS-WAHO-BioversityInternational (2012). Ainsi ils sont diversement valorisés pour leur intérêt alimentaire (Kouebou et al., 2006, 2008, 2013) mais également thérapeutique et cosmétique En effet les huiles essentielles et huiles végétales extraites de fruits (pulpes et/ou graines) rentrent dans diverses formulations médicamenteuses sous forme de poudre ou de lotion à l'exemple du fruit du baobab dont la coque réduite en poudre, est utilisée comme succédané du sel ou du tabac à priser (Eyog et al., 2006). C'est également le cas du beurre de karité, précieux pour le massage des nourrisson tandis que son savon évite la déshydratation de la peau (Francois et al., 2009). Ce beurre est fréquemment utilisé dans l'industrie cosmétique et en pharmacie pour la fabrication des pommades, des savons, des rouges à lèvre et des cataplasmes. Dans les savanes, le jus des amandes est réputé être un antivenimeux et soigne les diarrhées 
sanguinolentes (Malgras, 1992). Les graines de Carapa procera servent en cosmétique et pharmacologie (N'Klo, 2001). Excellents vermifuges, ces graines sont également utilisées comme adjuvants pour certains médicaments traditionnels. L'huile des graines est purgative, fébrifuge et soigne les rhumatismes (Malgras, 1992). Seulement une forte saisonnalité caractérise la quasi-totalité de ces fruits sauvages (Vivien \& Faure, 1995 ; Eyog et al., 2006, Aboubakar et al., 2009). Face à la demande grandissante, les programmes de sélection variétale en vue d'un accroissement de la productivité ne représentent qu'une partie de la solution. Les agents économiques (agriculteurs, producteurs semenciers, transformateurs, restauratrices et autres consommateurs) doivent disposer d'un marché pour offrir et/ou acquérir leurs produits. Les intervenants (étatiques ou privés) de la production et de la transformation doivent ajuster leurs activités afin de répondre à la demande croissante et aux besoins vitaux des consommateurs. Mais comment produire durablement et dynamiser le secteur fruitier dans un contexte général de dépréciation grandissante du pouvoir d'achat des agents économiques (Folefack, 2010a,b)? De plus en plus, la collecte et la vente des produits (plants, fruits et dérivés) sont des activités génératrices de revenu contribuant à l'approvisionnement en denrées et autres bien de

\section{MATÉRIELS ET MÉTHODES}

Site de l'étude et plan d'échantillonnage des fruits : Cette étude a été réalisée dans le Département de la Bénoué (Région du Nord, Cameroun) entre Juillet et Septembre 2012 (saison de pluie). Les entretiens avec les personnes ressources du ministère de l'agriculture, des experts du conservatoire fruitier de l'IRAD (Institut de Recherche Agricole pour le Développement) et des observations préliminaires sur les lieux de ventes urbains ont permis d'identifier les sites de l'étude. II s'agit de 5 marchés à vivres urbains et 2 marchés à vivres périurbains (Ouro Labbo et Takasko) des Arrondissements de Garoua. L'échantillonnage des fruits a été conduit en deux passages dans chacun de ces marchés. Le premier passage a servi à l'identification des acteurs en termes d'effectifs, de point de ventes, de volume et de prix des fruits commercialisés. Au second passage, les fruits ont été achetés en lots suivant selon un échantillonnage stratifié. premières nécessités (Eyog et al., 2006). Ceci est valable pour les populations démunies mais également pour les divers acteurs locaux notamment les propriétaires de vergers fruitiers, les producteurssemenciers et autres artisan(e)s de la transformation et du commerce informel. Dans le sahel en proie aux changements environnementaux et à la chaleur, la valeur socio-économique des fruits et dérivés (boissons chaudes ou rafraîchissantes) est considérable aussi bien au niveau national que régional (Temple et al., 1999 ; Eyog et al., 2006 ; Aboubakar et al., 2009). Seulement la rareté de statistiques fiables sur ce domaine concoure à la méconnaissance et à la sous-estimation du potentiel actuel des produits fruitiers dans la sécurité alimentaire. Cette situation justifie la présente étude dont le but est de contribuer à une connaissance de la biodiversité et valeur alimentaire des fruits au Cameroun. Après un échantillonnage des fruits commercialisés à Garoua (Région du Nord), l'étude à consisté en une identification et nomenclature des fruits sur la base de la littérature existante et sur une validation des botanistes et experts en agroforesterie de I'IRAD. Ensuite ont été appréciées les caractéristiques physico-sensorielles et marchandes des fruits disponibles à Garoua, principale métropole du nord-Cameroun.

Identification et nomenclature des fruits : L'identification et la nomenclature des fruits a été entamée sur la base de la littérature existante sur les fruitiers comestibles du Cameroun (Temple, 1999 ; Eyog et al., 2006) en accord avec les équipes du Programme Agroforesterie et fruitier de l'IRAD. L'Herbier National du Cameroun (rattaché à l'IRAD) a également été sollicité dans l'identification de ces fruits commercialisés dans cette région sahélienne. Ainsi ont pu être identifié les familles botaniques la nomenclature scientifique des ressources agricoles concernées autant que les noms des fruits en Français et en Fulfulde, les langues commerciales.

Caractérisation physico-sensorielle et marchande des fruits : Les masses du fruit entier, de sa pellicule et du noyau ont été relevées à l'aide d'une balance de précision (ACCULAB Sartorius Group, VIC- 303, USA). La pellicule et le noyau ont été pesés après une 
séparation manuelle, à l'aide d'un couteau et d'une spatule. Les portions comestibles et non comestibles ont été déduites par une différence arithmétique et exprimée en gramme et en pourcentage. Les opérations ont été répétées $(n \geq 5)$ suivant la nature des fruits. La forme, les couleurs du fruit et la nature (humide ou sèche) des fruits ont été notées par observation directe et par des références photographiques (Samsung, SGH-D780, Korea). Le goût du fruit a également été relevé après dégustation de la portion comestible. Les prix de vente

\section{RÉSULTATS ET DISCUSSION}

Diversité et nomenclature des arbres et fruits : Cette étude a recensé une vingtaine de fruits exposés à la vente sur les marchés de Garoua (tableau 1, figure 1) et appartenant à 14 familles botaniques dominées par les (en tasse, en tas ou en fruit individuel) ont été relevés lors de l'échantillonnage.

Analyse statistique : Pour les caractéristiques physiques des fruits, les résultats exprimés représentent la moyenne \pm écart type de 4 à 6 répétitions. Les analyses statistiques ont consisté en une analyse de variance (ANOVA) et une classification des moyennes (Multiple Range Test, 95 percent LSD) à l'aide du logiciel Stat Graphic Centurion XV (2005) au seuil de signification de $5 \%$.

Rutacées (agrumes). Leurs appellations en langues commerciales (Fulfulde et Français) sont assez proches de celles décrites par Eyog et al. (2006).

Tableau 1 : Noms des ressources et fruits représentés sur les marchés de Garoua

\begin{tabular}{|c|c|c|c|c|}
\hline \multicolumn{3}{|c|}{ Ressources } & \multicolumn{2}{|c|}{ Fruits } \\
\hline Nom & Famille & Nom scientifique & Nom en français & Nom en Fulfulde \\
\hline Ananas & Bromeliacees & Ananas comosus & Ananas & Ananas \\
\hline Pommier & Annonaceae & Annona squamosa & Pomme & Pomme \\
\hline Mandarinier & Rutaceae & Citrus reticulata & Mandarine & Mandarine \\
\hline Pamplemoussier & Rutaceae & Citrus maxima & Pamplemousse & Pamplemousse \\
\hline $\begin{array}{l}\text { Bananier- } \\
\text { Plantain }\end{array}$ & Musaceae & Musa sp. & Plantain & Plantain \\
\hline Oranger & Rutaceae & Citrus sinensis & Orange & Lemou \\
\hline Bananier & Musaceae & Musa sapientium L. & Banane & Nkodon \\
\hline Avocatier & Lauracees & Persea americana & Avocat & Phiyo \\
\hline Manguier & Anacardiceae & Manguifera indica & Mangue & Mangoro \\
\hline Citronnier & Rutaceae & Citrus limon & Citron & LemouLammoudoum \\
\hline Tamarinier & Caesalpiniaceae & Tamarindus indica Linn & Tamarin & Jabbe (Dabe) \\
\hline Baobab & Bombacaceae & Adansonia digitata Linn. & Fruit du baobab & Mboye (Boki) \\
\hline Faux Dattier & Palmaceae & Phoenix reclinata Jacq. & Datte & Dibinodje (Dibinohi) \\
\hline Jujubier Sauvage & Rhamnaceae & Ziziphus mauritania Lam. & Jujube sauvage & Djaabe (Djaabe) \\
\hline Jujubier & Rhamaceae & Ziziphus mauritania Lam. & Jujube & Kournadje \\
\hline $\begin{array}{l}\text { Palmier Ronier } \\
\text { n.d. }\end{array}$ & $\begin{array}{l}\text { Palmaceae } \\
\text { n.d. }\end{array}$ & $\begin{array}{l}\text { Borassus aethiopumMart. } \\
\text { [Borassus aethiopumMart.] }\end{array}$ & $\begin{array}{l}\text { Fruit du rônier } \\
\text { n.d. }\end{array}$ & $\begin{array}{l}\text { Doubbe (Doubi) } \\
\text { Goriba }\end{array}$ \\
\hline Nere & Mimisaceace & $\begin{array}{l}\text { Parkia biglobosa (Jacq.) } \\
\text { Benth. }\end{array}$ & Fruit du Nere & Naaredje \\
\hline n.d. & n.d. & n.d. & n.d. & Conkedje \\
\hline n.d. & n.d. & n.d. & n.d. & JabbeWaadou \\
\hline Karite & Sapotaceae & $\begin{array}{l}\text { Vitellaria paradoxa Gaerth. } \\
\text { C.F. }\end{array}$ & Fruit du karite & Caredje (Karehi) \\
\hline Prunier noir & Verbenaceae & Vitex doniana Sweet & Prune noir & Ngalbidje (Galbiki) \\
\hline $\begin{array}{l}\text { [Raisin du } \\
\text { gabon] }\end{array}$ & Anacardiaceae & $\begin{array}{l}\text { [Trchoscypha acuminata } \\
\text { Engl.] }\end{array}$ & [Raisin du gabon] & Tourssoudjé \\
\hline Dattier du désert & Balanitaceae & $\begin{array}{l}\text { Balanites aegyptiaca (Linn.) } \\
\text { Del. }\end{array}$ & Datte du désert & Tanne (Tani) \\
\hline
\end{tabular}

Les noms entre crochets [ ] sont en cours de vérification auprès de l'Herbier National du Cameroun.

Les mots entre parenthèses sont les noms en Fulfulde donnés par Eyog-Matig et al. (2006). 


\section{n.d. : en cours de détermination}
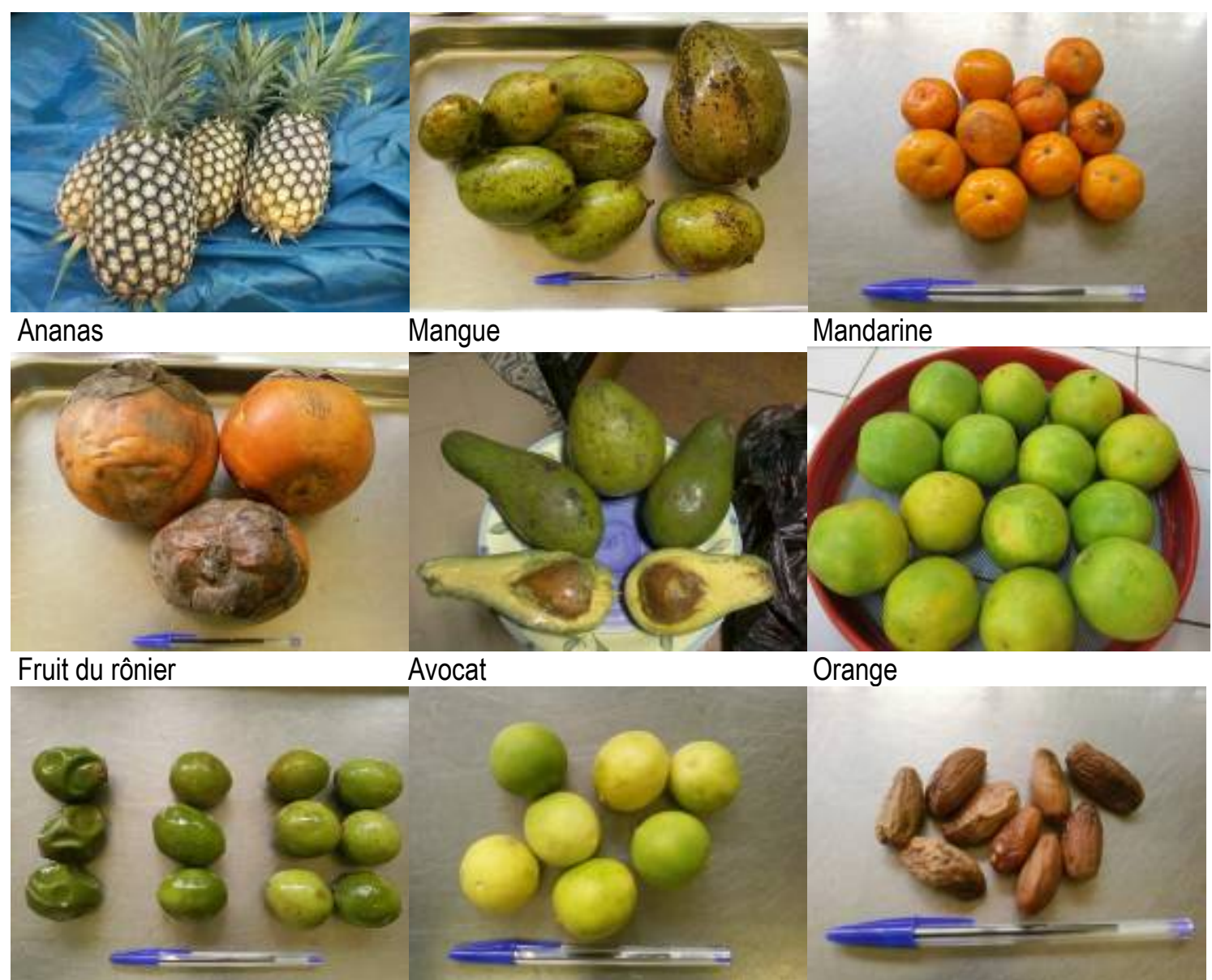

Avocat

Orange

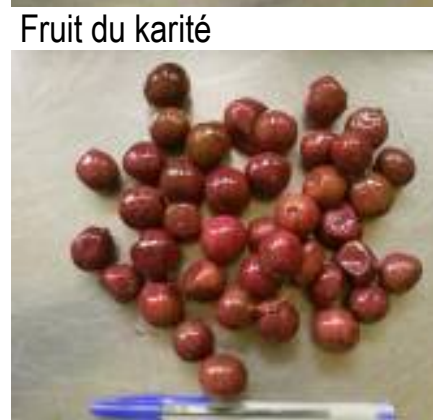

Citron
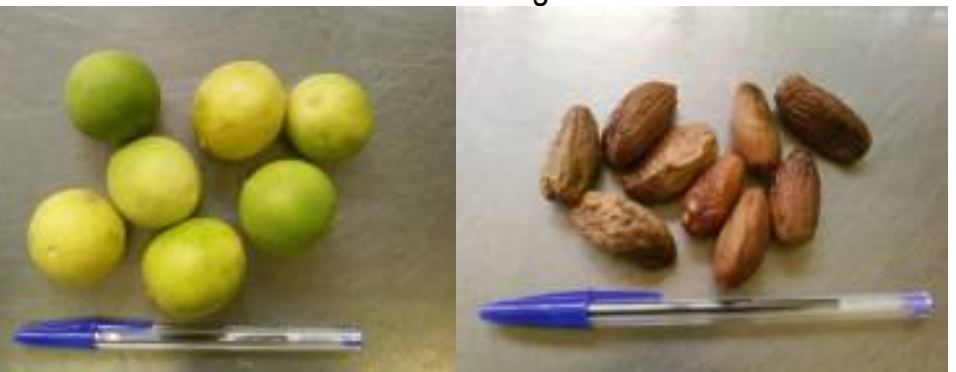

Raisin du Gabon

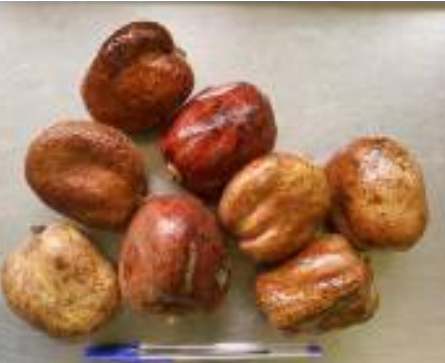

Datte (fruit du faux dattier)

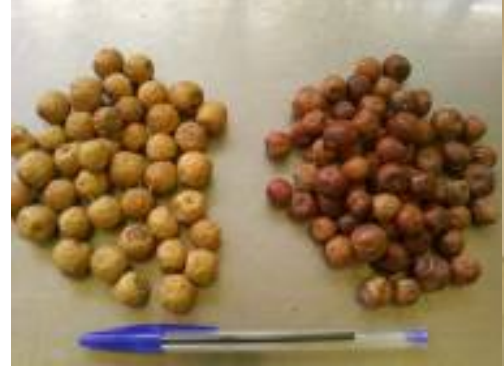

Goriba

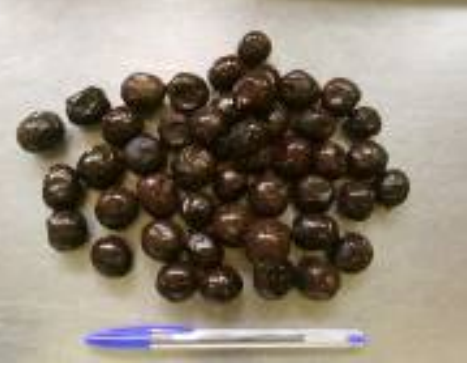

Jujube Jujube sauvage

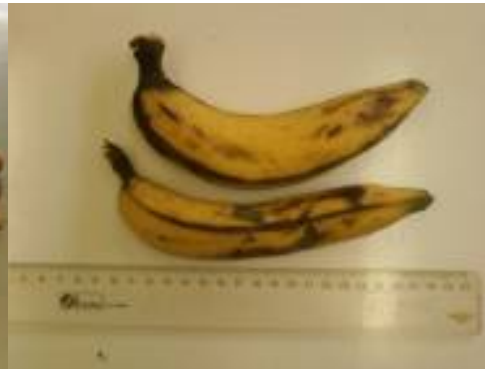

Plantain

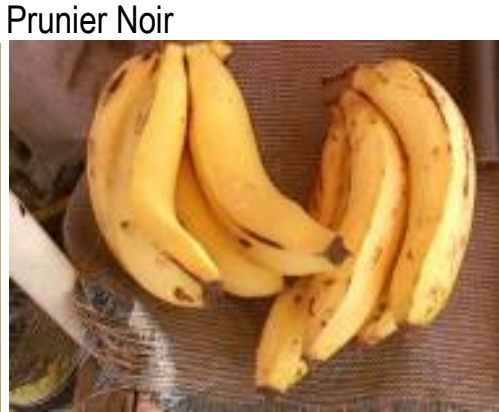

Banane

Figure 1 : Fruits en vente sur les marchés de Garoua 


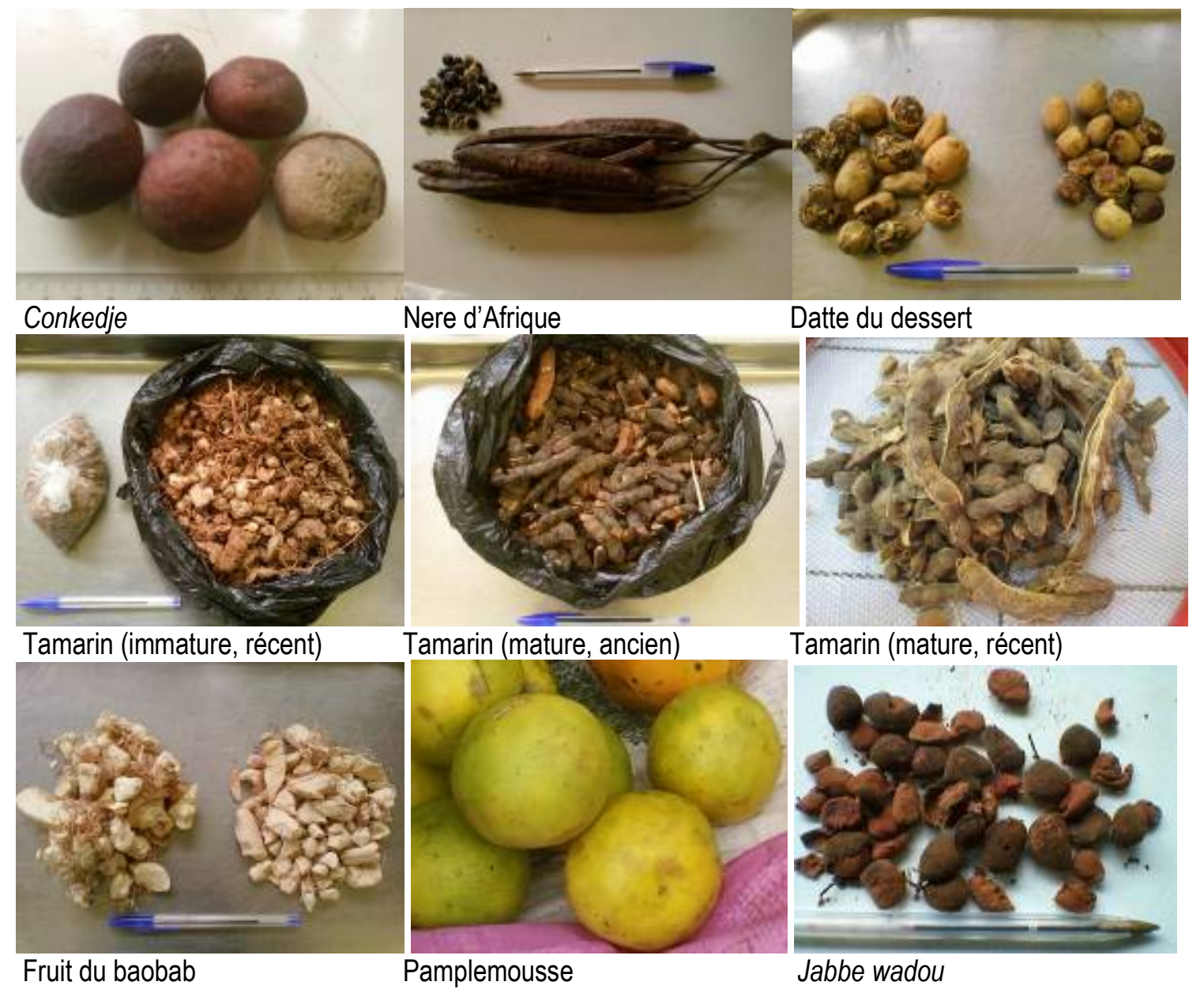

Figure 1 (suite) : Fruits en vente sur les marchés de Garoua

Tableau 2 : Distribution des fruits selon les zones et les points de vente dénombrés

\begin{tabular}{|c|c|c|c|c|c|c|c|c|c|}
\hline \multirow[t]{2}{*}{ Fruit $^{*}$} & \multicolumn{5}{|c|}{ Zone urbaine } & \multicolumn{2}{|c|}{ Zone péri-urbaine } & \multirow[t]{2}{*}{ Total } & \multirow[t]{2}{*}{$\%$} \\
\hline & Zone 1 & Zone 2 & Zone 3 & Zone 4 & Zone 5 & Ouro Labbo & Takasko & & \\
\hline Ananas & 5 & 2 & 2 & 0 & 0 & 0 & 0 & 9 & 2,61 \\
\hline Avocat & 27 & 17 & 1 & 0 & 0 & 6 & 3 & 54 & 15,65 \\
\hline Banane & 9 & 6 & 8 & 0 & 0 & 2 & 6 & 31 & 8,99 \\
\hline Baobab & 6 & 1 & 1 & 0 & 0 & 7 & 1 & 16 & 4,64 \\
\hline Citron & 2 & 2 & 3 & 0 & 2 & 6 & 4 & 19 & 5,51 \\
\hline Conkedje & 0 & 0 & 0 & 0 & 0 & 0 & 2 & 2 & 0,58 \\
\hline Faux dattier & 4 & 1 & 0 & 0 & 0 & 1 & 1 & 7 & 2,03 \\
\hline Datte du désert & 4 & 0 & 0 & 0 & 0 & 0 & 1 & 5 & 1,45 \\
\hline Fruit du karité & 0 & 0 & 0 & 0 & 0 & 9 & 17 & 26 & 7,54 \\
\hline Goriba & 1 & 0 & 0 & 0 & 0 & 3 & 3 & 7 & 2,03 \\
\hline Jujubier sauvage & 3 & 0 & 0 & 0 & 0 & 3 & 1 & 7 & 2,03 \\
\hline jujube & 3 & 0 & 0 & 0 & 0 & 2 & 1 & 6 & 1,74 \\
\hline Mandarine & 1 & 0 & 0 & 0 & 0 & 0 & 0 & 1 & 0,29 \\
\hline Mangue & 26 & 5 & 4 & 4 & 0 & 0 & 7 & 46 & 13,33 \\
\hline Fruit du néré & 0 & 0 & 0 & 0 & 0 & 0 & 1 & 1 & 0,29 \\
\hline Prunier noir & 0 & 0 & 0 & 0 & 0 & 1 & 3 & 4 & 1,16 \\
\hline Orange & 10 & 2 & 3 & 0 & 0 & 1 & 3 & 19 & 5,51 \\
\hline
\end{tabular}




\begin{tabular}{llllllllll} 
Pamplemousse & 1 & 1 & 0 & 0 & 0 & 0 & 0 & 2 & 0,58 \\
Plantain & 1 & 0 & 5 & 0 & 0 & 0 & 0 & 6 & 1,74 \\
Pomme & 4 & 1 & 0 & 0 & 0 & 0 & 0 & 5 & 1,45 \\
Tamarin & 11 & 5 & 2 & 0 & 6 & 20 & 23 & 67 & 19,42 \\
Raisin du Gabon & 0 & 0 & 0 & 0 & 0 & 2 & 3 & 5 & 1,45 \\
\hline Total & 118 & 43 & 29 & 4 & 8 & 63 & 80 & 345 & 100 \\
$\%$ & 34,20 & 12,46 & 8,41 & 1,16 & 2,32 & 18,26 & 23,19 & 100 & \\
\hline
\end{tabular}

* Les noms en italique sont en cours d'identification

Tableau 3 : Diversité des variétés fruitières disponibles au conservatoire de l'IRAD à Garoua

\begin{tabular}{|c|c|c|c|c|c|}
\hline \multicolumn{2}{|c|}{ Agrumes } & \multicolumn{4}{|c|}{ Manguiers } \\
\hline Variétés & Nombre & Variétés & Nombre & Variétés & Nombre \\
\hline Clémentine & 6 & Alphonse Hawaï & 1 & Amélie & 6 \\
\hline Mandarine & 31 & Auguste & 4 & Baratier & 1 \\
\hline Satsuma & 16 & Beverley & 8 & Brook's & 5 \\
\hline Tangor & 5 & Cambodiana & 3 & Camayenne & 2 \\
\hline Tangelo & 8 & Coq's Hall & 4 & Davis Haden & 5 \\
\hline Orange & 18 & Divine & 2 & Diibelor Casamance & 1 \\
\hline Pamelo & 11 & Early Gold & 1 & Edward's & 6 \\
\hline Pamplemousse & 4 & Eldon & 10 & Florigon & 3 \\
\hline Lime & 4 & Francis & 4 & Julie & 5 \\
\hline Citron & 7 & Glazier Mali & 2 & Haden & 5 \\
\hline Cédrat & 2 & Indi-Bishinara & 2 & |fac $\mid$ & 3 \\
\hline Combavat & 1 & Irwin & 9 & Julie Kassowa & 6 \\
\hline Bigaradier (fleur) & 2 & julie Nyombe & 7 & Keith & 5 \\
\hline Calamondin & 1 & Kensington & 6 & Kent & 7 \\
\hline Kumquat & 3 & Lippens & 3 & Malembe & 3 \\
\hline Lime quat & 2 & Mangotine & 2 & Maya & 2 \\
\hline Hybride de prospection & 1 & Missinglio & 2 & Palmer & 13 \\
\hline & & Passy Hative & 3 & Peter Passant & 2 \\
\hline & & Romanea & 2 & Ruby & 34 \\
\hline & & Sabot & 8 & Sabre & 2 \\
\hline & & Sans Pareille & 1 & Sensation & 3 \\
\hline & & Smith & 7 & Springfield/Zill & 4 \\
\hline & & Tommy Atkins & 9 & Tommy Atkins Mali & 4 \\
\hline & & Valencia & 3 & Victoria & 2 \\
\hline & & Xio-Cat-Mytho & 2 & Yellow & 2 \\
\hline & & Zill & 20 & Anderson & 1 \\
\hline & & Grosse Rouge & 1 & Maroua I & 1 \\
\hline & & Maroua II & 3 & Maroua III & 1 \\
\hline & & N'gaoundere & 15 & Punda de la Muna & 4 \\
\hline & & Carmen & 1 & Dikeson & 3 \\
\hline & & Gomera I & 1 & Gomera II & 1 \\
\hline & & Gomera III & 2 & Osteint & 3 \\
\hline & & Dike & 3 & Sun-set & 1 \\
\hline & & Djibelor & 2 & Boy-Toy & 1 \\
\hline & & Tolber & 4 & Gleen & 3 \\
\hline & & Soudan & 1 & & \\
\hline Total & 122 & Total & 158 & Total & 150 \\
\hline
\end{tabular}


Le tableau 2 montre leur répartition et fréquence suivant les points de vente dénombrés à travers les 7 marchés considérés. Ainsi 345 lots (équivalent aux points de vente) ont été recensés et laissent apparaître trois groupes rangés selon l'abondance des points de vente. Les fruits du rônier et « Jabbé wadou » rencontrés chez un seul vendeur n'ont pas été pris en compte dans ses statistiques. Les deux groupes majoritaires sont constitués de 8 fruits classés ainsi qu'il suit : - 1ergroupe (7,54 à 19,42\% des lots) : Avocat, Banane, Fruit de karité, Mangue, Tamarin ; - 2e groupe (4,64 à 5,51\% des lots) : Baobab, Citron, Orange. En milieu urbain la zone 1 contient le plus important regroupement de commerçants fruitiers $(34,20 \%)$ il est secondé par le marché péri-urbain de Takasko (23,19\%) puis celui de Ouro Labbo (18,26\%). Ces données montrent l'importance des zones urbaines et peri-urbaines dans la vente fruits. II existerait quelques spécificités géographiques étant donné que les avocats, oranges et mangues sont d'avantages représentés en zone urbaine, contrairement au fruit du karité et tamarin. Ceci serait du au fait que les premiers proviennent $d u$ commerce de gros depuis les marchés de Ngaoundéré, du Sud -Cameroun ou du Nigéria (Aboubakar et al., 2009), alors que les seconds davantage collectés en zone rurale (Kater et al., 1992 ; El-Siddig et al., 2006 ; Bourou et al., 2010) seraient acheminés en petit lots sur les divers marché péri-urbains et ruraux. La biodiversité des agrumes et manguiers proviendrait au moins en partie du conservatoire fruitier de l'IRAD à Kismatari (Arrondissement de Garoua $3^{\mathrm{e}}$ ). En effet, une collection commerciale et semencière d'agrumes et de manguiers et d'autres espèces (palmier dattier, raisin de table, espèces sous-utilisées), y ont été introduites à partir de 1983. Le tableau 3 présente les effectifs initiaux (430 pieds) pour les différentes espèces $(n=17)$ d'agrumes (122 variétés) ainsi que les manguiers (71 variétés) également conservés pour des besoins de Recherche d'adaptation et développement (Normand, 1992). La pépinière de Kismatari est dotée d'une capacité de production de 20000 plants toutes espèces confondues. Le régime de production des plants dans les années 90 était de 10000 plants par an, dont 6000 agrumes (Normand, 1992).

Caractéristiques physico-sensorielles des fruits : Les tableaux 4 et 5 indiquent les caractéristiques physique et sensorielle des fruits. Quatre formes rangées par ordre décroissant d'importance ainsi que suit : fruits sphérique, ovoïde, ovale, en gousse et allongée. Leurs pellicules présentent 5 couleurs : marron, Verdâtre, Rouge violacée, Orange et Noir (figure 1). Les parties comestibles sont davantage colorées (8 couleurs) et se présent ainsi que suit : pulpes beige, marron, jaunâtre, verdâtre, blanchâtre, rouge violacée, orange et noire. Selon leur nature les fruits sont sec ou humide tandis que leur goût varie avec une domination de la saveur sucrée, puis acide et fade. Ces données sont proches de celle décrites par la littérature (Arbonnier, 2000, Eyog et al., 2006). Plusieurs groupes de fruits peuvent se distinguer $(P<0,05)$ en fonction de la portion comestible : $1^{\text {er }}$ groupe étant constitué de l'avocat $(122,18 \mathrm{~g})$ et de la mangue $(121,4 \mathrm{~g})$ alors que le dernier groupe est constitué du Jabbewadou $(0,19 \mathrm{~g})$, du jujube sauvage $(0,3 \mathrm{~g})$, du tamarin $(0,56 \mathrm{~g})$ et du jujube $(0,6 \mathrm{~g})$. Ces fruits ont ainsi entre $24,14 \%$ (Conkedje) et 70,47\% (banane) de portion comestibles.

Ces valeurs de portion comestible se rapprochent de celles FAO/INFOODS/WAHO/Bioversity International (2012) en ce qui concerne la mangue $(71 \%)$, la banane $(64 \%)$, et le tamarin $(47 \%)$ contrairement à l'orange $(73 \%)$, le karité $(100 \%)$, le fruit du baobab $(28 \%)$, le citron $(64 \%)$, les dattes séchées $(100 \%)$, le jujube sauvage (93\%). Des différences qui peuvent se justifier par la nature des fruits considérés. En effet la présente étude se base sur les fruits échantillons tels qu'exposés à la vente alors que la seconde semble révéler les valeurs pour les fruits plus ou moins nature.

Tableau 4 : Caractéristiques sensorielle et marchande des fruits

\begin{tabular}{llllll}
\hline \multirow{2}{*}{ Fruits } & \multicolumn{2}{c}{ Caractéristiques sensorielles } & \multicolumn{2}{c}{ Caractéristiques de vente } \\
\cline { 2 - 6 } & Couleur de la pulpe & Nature & Goût & Prix (FCFA) & Unité \\
\hline Ananas & Beige à jaunâtre & Humide & Sucré/acide & $500-1300$ & Fruit \\
Avocat & Vert & Humide & Fade & $100-250$ & Fruit \\
Banane & Beige à Jaunâtre & Humide & Sucré & $50-100$ & Fruit \\
Fruit de karité & Verdâtre & Humide & Sucré & $50-100$ & Tas \\
Citron & Jaunâtre & Humide & Acide & $10-25$ & Fruit \\
Conkedje & Verdâtre & Sèche & Sucré & $25-50$ & Tas \\
Goriba & Marron clair & Sèche & Sucré & $20-25$ & Fruit \\
Jujube sauvage & Marron & Sèche & Sucré & $25-50$ & Sachet \\
Jujube & Marron clair & Sèche & Sucré (amère) & $50-125$ & Sachet \\
\hline
\end{tabular}




\begin{tabular}{llllll}
\hline Mangue & Jaunâtre a orangée & Humide & Sucré/acide & $25-100$ & Fruit \\
Fruit du néré & Jaune oranger & Sèche & Sucré & $50-100$ & Tas \\
Prunier noir & Noir & Humide & Sucré & $50-100$ & Tas \\
Orange & Jaunâtre & Humide & Sucré/acidulée & $25-100$ & Fruit \\
Tamarin & Marron clair a fonce & Sèche & Acide & $50-100$ & Sachet \\
Datte du dessert & Marron orangé & Sèche & Sucré (amère) & $25-50$ & Sachet \\
Faux Dattier & Blanchâtre & Sèche & Sucré & $50-100$ & Sachet \\
Jabbewadou & Marron & Sèche & Sucré/Acidulé & 50 & Sachet \\
Fruit du Baobab & Orange & Sèche & Sucré/Acidulé & $25-50$ & Sachet \\
Raisin du Gabon & Rouge violacé & Humide & Sucré & $50-100$ & Tas \\
Mandarine & Beige à oranger & Humide & Sucré/acide & $25-50$ & Fruit \\
Fruit du rônier & Orange & Humide & Sucré & 50 & Fruit \\
Pamplemousse & Jaunâtre & Humide & Sucré (amère) & $50-100$ & Fruit \\
Pomme & Blanchâtre & Humide & Sucré & $250-300$ & Fruit \\
Plantain & Beige à Jaunâtre & Humide & Sucré/fade & $250-500$ & Tas \\
\hline
\end{tabular}

Tableau 5 : Caractéristiques physiques et portion comestible des fruits

\begin{tabular}{|c|c|c|c|c|c|c|}
\hline Fruits & Forme & $\begin{array}{l}\text { Poids } \\
\text { entier }(\mathrm{g})\end{array}$ & $\begin{array}{l}\text { Pellicule } \\
\text { (g) }\end{array}$ & $\begin{array}{l}\text { Noyau+rejet } \\
\text { intérieur (g) }\end{array}$ & $\begin{array}{l}\text { Portion comesti } \\
\text { (g) }\end{array}$ & $\begin{array}{l}\text { ible } \\
(\%)\end{array}$ \\
\hline Avocat & Ovoïde & $216,90^{\mathrm{h}} \pm 31,5$ & $43,4^{d} \pm 5,0$ & $51,3^{f} \pm 2,4$ & $122,18^{f} \pm 25,52$ & 56,34 \\
\hline Banane & Allongé & $96,27^{d} \pm 2,81$ & $28,32^{c} \pm 1,27$ & $0,00^{\mathrm{a}} \pm 0,00$ & $67,84^{d} \pm 2,08$ & 70,47 \\
\hline Fruit du karité & Ovoïde & $48,20^{c} \pm 2,06$ & $6,86^{b} \pm 2,67$ & $20,41^{d} \pm 2,41$ & $20,90^{b c} \pm 2,8$ & 43,36 \\
\hline Citron & Sphérique & $39,86^{b c} \pm 5,01$ & $6,94^{b} \pm 1,62$ & $14,45^{c} \pm 1,02$ & $18,48^{a b c} \pm 2,78$ & 46,37 \\
\hline Conkedje & Ovoïde & $17,40^{\mathrm{ab}} \pm 0,82$ & $4,19^{\mathrm{ab}} \pm 0,15$ & $8,97^{b} \pm 0,43$ & $4,25^{\mathrm{ab}} \pm 0,75$ & 24,14 \\
\hline Goriba & Ovoïde & $91,26^{d} \pm 12,07$ & $7,18^{b} \pm 1,14$ & $53,91^{f} \pm 6,63$ & $30,18^{c} \pm 6,53$ & 33,08 \\
\hline Jujube sauvage & Sphérique & $0,92^{\mathrm{a}} \pm 0,11$ & $0,26^{a} \pm 0,05$ & $0,36^{a} \pm 0,03$ & $0,30^{a} \pm 0,07$ & 33,33 \\
\hline Jujube & Sphérique & $1,51^{a} \pm 0,11$ & $0,26^{a} \pm 0,09$ & $0,66^{a} \pm 0,10$ & $0,60^{a} \pm 0,07$ & 40,00 \\
\hline Mangue & Ovoïde & $191,309 \pm 53,16$ & $41,85^{d} \pm 1,67$ & $28,05^{\mathrm{e}} \pm 1,86$ & $121,40^{f} \pm 51,42$ & 63,46 \\
\hline Fruit du néré & Gousse & $10,75^{a} \pm 5,38$ & $4,13^{a b} \pm 1,88$ & $2,00^{\mathrm{a}} \pm 1,40$ & $4,62^{a b} \pm 2,43$ & 42,59 \\
\hline Prunier noir & Sphérique & $7,10^{\mathrm{a}} \pm 0,77$ & $1,11^{a} \pm 0,22$ & $2,77^{a} \pm 0,36$ & $3,23^{a b} \pm 0,55$ & 45,07 \\
\hline Orange & Sphérique & $155,21^{f} \pm 4,68$ & $30,74^{\mathrm{c}} \pm 3,55$ & $54,07^{\dagger} \pm 5,92$ & $70,40^{\text {de }} \pm 5,90$ & 45,36 \\
\hline Tamarin & Gousse & $1,41^{a} \pm 0,3$ & $0,44^{a} \pm 0,15$ & $0,42^{\mathrm{a}} \pm 0,11$ & $0,56^{a} \pm 0,2$ & 39,72 \\
\hline Datte du désert & Ovale & $4,82^{\mathrm{a}} \pm 1,72$ & $1,02^{a} \pm 0,33$ & $2,21^{a} \pm 0,97$ & $1,60^{\mathrm{ab}} \pm 0,47$ & 33,20 \\
\hline Raisin du Gabon & Sphérique & $6,01^{a} \pm 1,38$ & $2,20^{\mathrm{ab}} \pm 0,45$ & $1,60^{\mathrm{a}} \pm 0,48$ & $2,20^{\mathrm{ab}} \pm 0,49$ & 36,61 \\
\hline Jabbewadou & Ovoïde & $0,71^{a} \pm 0,07$ & $0,27^{\mathrm{a}} \pm 0,04$ & $0,25^{a} \pm 0,08$ & $0,19 \mathrm{a} \pm 0,11$ & 26,76 \\
\hline Faux dattier & Ovale & $4,95^{a} \pm 0,82$ & $0,00^{\mathrm{a}} \pm 0,00$ & $1,67^{\mathrm{a}} \pm 0,21$ & $3,28^{\mathrm{ab}} \pm 0,74$ & 66,26 \\
\hline Fruit du baobab & Ovale/grappe & $93,92^{d} \pm 7,42$ & $0,00^{\mathrm{a}} \pm 0,00$ & $61,15^{g} \pm 7,72$ & $32,7^{c} \pm 5,79$ & 34,89 \\
\hline Plantain & Allongé & $141,36 f \pm 27,66$ & $49,51 \mathrm{e} \pm 14,32$ & $0,00 a \pm 0,00$ & $89,36^{\mathrm{e}} \pm 14,91$ & 63,21 \\
\hline Ananas & Ovale & n.d. & n.d. & n.d. & n.d. & n.d. \\
\hline Pomme & Sphérique & n.d. & n.d. & n.d. & n.d. & n.d. \\
\hline pamplemousse & Sphérique & n.d. & n.d. & n.d. & n.d. & n.d. \\
\hline Mandarine & Sphérique & n.d. & n.d. & n.d. & n.d. & n.d. \\
\hline Fruit du rônier & Ovoïde & n.d. & n.d. & n.d. & n.d. & n.d. \\
\hline
\end{tabular}

n.d., non-déterminé

Les moyennes de la même colonne ayant les exposants différents sont significativement différentes à $P \leq 0,05$ selon le test de classification selon la plus petite différence significative (95 percent LSD).

Caractéristiques marchandes des fruits : Le tableau 4 donne également les prix et unité de vente des fruits. Les fruits sont vendus sous diverses formes allant de l'échantillon unitaire (1 fruit) au lot (sachet ou tas) en fonction de l'espèce et du vendeur. Les prix sont très variables et compris entre 10 francs CFA l'unité de citron à 1300 francs CFA l'ananas. Ces prix sont assez comparable à ceux précédemment publiés au Cameroun 
(Eyog-Matig et al., 2006). Trois groupes de fruits peuvent se distinguer en fonction de prix : 1 er groupe (environ 500 $\mathrm{Frs} / \mathrm{kg}$ ) : la banane, le baobab, le citron, le jujube et la mandarine; $2^{\mathrm{e}}$ groupe (200 à $300 \mathrm{Frs} / \mathrm{kg}$ ) : la datte du

\section{CONCLUSION ET PERSPECTIVES}

La consommation des fruits est importante dans la nutrition humaine d'autant plus que pour les populations vulnérables des zones subsahariennes semi-arides, il s'agit de ressources de subsistance. Seulement le manque de statistiques fiables sur ces ressources concoure à limiter leur protection tandis que la rareté des données nutritionnelles sur les fruits serait une entrave à leur valorisation alimentaire. De plus en plus un consensus émerge en faveur de l'amélioration de la consommation des fruits à travers la promotion de l'arboriculture fruitière mais également à partir des produits de cueillette. Ainsi des observations préliminaires ont été initiées dans le Département de la Bénoué (Région du Nord, Cameroun) en vue d'apprécier la biodiversité et la valeur alimentaire des fruits. Une vingtaine de fruits ont été commercialisés sur les

\section{REMERCIEMENTS}

Les auteurs remercient I'IRAD (Station de Garoua) pour le soutien multiforme (financement partiel, accès à la documentation, assistance des experts des programmes sur les fruits et l'agroforesterie.). Mademoiselle Habiba Samira (stagiaire de l'IUT de Ngaoundéré) estégalement remerciée pour son implication à travers la collecte et la saisie des données des registres, d'enquêtes de terrain et

\section{RÉFERENCES}

Aboubakar D, Sorto M, Mbayabe L, Woin N, Bourou S, Gandebe M, 2009. Commercialisation des fruits dans les savanes d'Afrique centrale. Communication au Colloque "Savane Africaines en développement : innover pour durer », 20-24 avril 2009, Garoua, Cameroun. Livre de résumé de la communication, p 58.

Arbonnier M, 2000. Arbres, arbustes et lianes des zones sèches d'Afrique de l'Ouest. CIRAD-MNHNUICN, France, $541 \mathrm{p}$.

Bâ AM, Plenchette C, Danthu P, Duponnois R, Guissou $\mathrm{T}$, 2000. Functional compatibility of two arbuscular mycorrhizae with thirteen fruit trees in Senegal, Agroforestry System. 50: 95-105.

Bourou S, Ndiaye F, Diouf M, Van Damme P, 2010. Tamarind (Tamarindus indica L.) parkland désert, l'avocat, la mangue, le prunier noir, orange, tamarin, raisin du Gabon et, $3^{3}$ groupe (140 à $100 \mathrm{Frs} / \mathrm{kg}$ ) : la datte, le fruit du karité, le jujubier sauvage et le fruit du néré.

marchés de Garoua entre Juillet et Septembre. Les groupes majeurs sont issus de la savane sahélienne (fruit de karité, mangue, tamarin, baobab, citron) ou importés de la zone équatoriale (avocat, banane) voir du Nigéria (orange). Ils appartiennent à 14 familles botaniques dominées par les Rutacées (agrumes). Leur identité et les observations préliminaires sur leurs propriétés physico-sensorielle et marchande confirment la diversité des fruits autant que leur accessibilité au regard de leur prix de vente (10 à 100 francs CFA ( 0,05 à 0,5 USD) pour la plus part). Ces observations sont en cours dans d'autres zones et période de l'année. Elles serviront de base à l'élaboration d'une stratégie de la recherche en vue de la promotion (via la vente, la transformation et la consommation) des ressources fruitières au Cameroun.

d'analyse de laboratoire. Les auteurs sont enfin reconnaissants aux commerçants des marchés visités ainsi que les autres facilitateurs locaux durant les descentes sur les marchés urbains et périurbains de Garoua.

mycorrhizal potential within three agroecological zones of Senegal. Fruits, vol. 65, 1-9.

De Caluwé E, Halamova K, Van Damme P, 2010. Tamarindus indica L. A Review of Traditional Uses, Phytochemistry and Pharmacology, $38 \mathrm{p}$.

El-Siddig K, Gunasena HPM, Prasad BA, Pushpakumara DKNG, Ramana KVR, Vijayand P, Williams JT, 2006. Tamarind (Tamarindus indica L.), Br. Libr., Southampt., U.K.126 p.

Eyog Matig O, Ndoye O, Kengue J, Awono A., (Editors), 2006. Les fruitiers forestiers comestibles du Cameroun. IPGRI (International Plant Genetic Ressources Institute). 204 p.

FAO/INFOODS/WAHO/Bioversity International, 2012. West African Food Composition Table - Table de composition des aliments d'Afrique de 
l'Ouest. The Food and Agriculture Organization of the United Nations, The International Network of Food Data Systems, The West African Health Organization, Bioversity International.

Folefack DP, 2010a. Coordination des acteurs dans un contexte de crise : le cas de la filière coton au Cameroun depuis 1990 - 2010. Thèse de Doctorat en économie, Université Rennes 2 Haute Bretagne, $332 \mathrm{p}$.

Folefack DP, 2010b. Poverty and share revenue in the Cameroon cocoa zone. Tropicultura 28 (1) 5-9.

Francois M, Niculescu N, Badini Z, Mamadou Diarra, 2009. Le beurre de karité au Burkina Faso: entre marché domestique et filières d'exportation. Cahier Agriculture, 18 (4) 369375.

Grivetti LE, 1978. Nutritional Success in a Semi-arid Land: Examination of the Eastern Kalahari, Botswana. Am. J. of Clinical Nutr. 31: 1204 1220.

Herzorg F, Farah Z, Amado R, 1994. Composition and Consumption of Gathered Wild Fruits in the VBaoule, Cote d' Ivoire. J. Ecol. Food and Nutr. 32 (3-4): 181 - 196.

Humphrey CM, Clegg MS, Keen CL, Grivetti LE, 1993. Food Diversity and Drought Survival. The Hausa Example. Int. J. of Food Sci. \& Nutr. 44: 1 - 16.

Kater JM, Kante S, Budelman A, 1992. Karité (Vitellaria paradoxa) and néré (Parkia biglobosa) associated with crops in Mali. Agroforestery Systems 18: 89-105

Kouebou CP, Essia Ngang J-J, Etoa F-X, 2008. Variation de qualité au sein des unités traditionnelles de transformation du maïs en farines, pâtes et Gaari. In « Agricultures et Développement Urbain en Afrique de l'ouest et du Centre ", édition L'Harmattan (France). pp 151-161.
Kouebou CP, Essia-Ngang J-J, Dzudie T, Mbofung CMF, Etoa F-X, 2006. Antimicrobial activities of Kilbu and tamarind pulp extracts used in traditional medicine and cereal gruel in Cameroon. International Journal of Tropical Medicine 1(4) 145-151.

Kouebou CP, Achu M, Nzali S, Machawe Chelea, Bonglaisin J, Kamda A, Djiele P, Yadang G, Ponka R, Ngoh Newilah G, Nkouam G, Teugwa C, Kana Sop MM, 2013. A review of composition studies of Cameroon traditional dishes: Macronutrients and minerals. Food Chemistry 140 (2013) 483-494.

Malgras D, 1992. Arbres et arbustes guérisseurs des savanes maliennes. Karthala, $478 \mathrm{p}$.

N'Klo 0, 2001. Situation des ressources génétiques forestières de la Côte d'lvoire en zone de savanes, FAO/IPGRI/ICRAF.

Normand $F, 1992$. Le conservatoire de matériel végétal agrumicole de Garoua, Nord Cameroun. Fruits, numero spécial agrumes, 1992, 151-156

Ogle BM and Grivetti LE, 1985. Legacy of the Chameleon: Edible and Wild Plants in the Kingdom of Swaziland, Southern Africa. A Cultural, Ecological and Nutritional Study. Part IV - Nutritional Analysis and Conclusions. J. Ecol. Food Nutr. 17: 41 -64.

Smith GC and Grivetti LE, 1994. Cultural Use of Edible Wild Plants in Burkina Faso, West Africa. FASEB J. 8 : A183.

Temple L, 1999. Le marché des fruits et légumes au Cameroun-quantification des flux-analyse des prix. IRAD/CIRAD. 158p.

Vivien and Faure JJ, 1995. Fruitiers sauvages d'Afrique - espèces du Cameroun. Editions NguilaKerou, France, 416p. 